\title{
Letters
}

\section{Attenuation of Dispersed Waves ${ }^{1}$}

\author{
L. Knopoft, K. AKI, C. B. Archambead, \\ A. Ben-Menahem, and J. A. Hudson \\ Seismological Laboratory \\ Califormia Institute of Technology, Pasadena
}

A measure of the absorption of elastic waves is the specific absorption coefficient $1 / Q$. In dispersive mediums, whether the dispersion is due to geometry, inhomogeneity, or both, measurements are often made outside the body and the measurements must be interpreted as to the distribution of values of $1 / Q$ within the body.

Two definitive experiments of this type are those performed using standing waves set up in a confined sample of the body and with waves that propagate through or on the surface of the body. Typical examples of these experiments involve the measurement of the damping coefficient of the free modes of vibration of the earth and the measurement of the attenuation factor of propagating surface waves on the earth. These two types of experiments can themselves be interpreted in terms of dimensionless attenuation factors. We call the dimensionless attenuation factors in the standing wave and propagating wave experiments $1 / Q_{r}$ and $1 / Q_{x}$, defined as the logarithmic decrements $\pi / Q_{T}$ and $\pi / Q_{x}$ in each experiment. Then in a damped standing wave the amplitude will diminish with time $t$ at a fixed point as $\exp \left(-\pi t / T Q_{T}\right)$, where $T$ is the period. In a propagating monochromatic wave the amplitude will diminish with distance $x$ as $\exp \left(-\pi x / c T Q_{x}\right)$, where $c$ is the phase velocity.

In general, the quantities $Q_{x}$ and $Q_{r}$ differ; the interpretation of these two quantities as to the distribution of the intrinsic $Q$ in the interior of a body may be made along different lines. However, they are related by

$$
U Q_{T}=c Q_{X}
$$

1 Contribution 1217, Division of Geological Sciences, California Institute of Technology, Pasadena. where $U$ is the group velocity. Brune [1962] has shown that (1) holds, although he has not used this notation. A new proof of the relationship is presented in this note.

We assume throughout this discussion that $\left|Q_{r, x}\right| \gg 1$ and that dispersion due to geometry is much stronger than dispersion associated with causal conditions derived from nonvanishing values of $1 / Q$; dispersion due to causality must be at the most of the order of $1 / Q$ for large $Q$ [Futterman, 1963].

It will be sufficient to demonstrate the relationship for a one-dimensional structure. Let a solution to the problem of wave propagation in an unbounded medium be exp $[i(\omega t-k x)]$, where $k$ is the wave number. Assume further that a relationship between $\omega$ and $k$ exists, derivable from the boundary conditions

$$
\omega=\omega\left(k ; p_{l}, q_{i}\right) \quad \begin{aligned}
& l=1,2, \cdots n \\
& j=1,2, \cdots m
\end{aligned}
$$

In (2) the $p$ 's and the $q$ 's are parameters of the problem (elastic constants, densities, thicknesses of layers, etc.).

The relationship (2) is assumed to be continuous and differentiable with respect to $k$ and the parameters. This assumption is valid even if the observations are made of the discrete spectrum only and even if the continuous spectrum is not observed over the entire frequency domain. A second relationship must be applied to (2) to obtain the standing wave solutions. As an example, we note that for a spherical body the eigenvalue equation (2) can be expressed in terms of a parameter which is the order of a spherical harmonic; this is a continuous function of the order, and the free 
modes are obtained only when the solution is restricted to integral values of order.

Let us first consider a model in which no dissipation exists anywhere; next we shall endow all or part of the same structure with intrinsic dissipation. The eigenvalue equation 2 in the lossless case is

$$
\omega_{0}=\omega\left(k_{0} ; p_{l 0}, q_{i 0}\right)
$$

the zero subscripts referring to the dissipationless mode; we consider only real roots to this equation. Thus $\omega_{0}$ is real for $k_{0}$ real; several roots to this equation may exist.

Now consider a model with a small amount of intrinsic dissipation. We let some of the parameters $p_{l}$, for example the elastic constants, be changed to the values

$$
\begin{aligned}
p_{l} & =p_{t 0}+i p_{l 1}(\omega) \quad l=1, \llbracket 2, \cdots \cdots n \\
\left|p_{l 1}\right| & \ll\left|p_{l 0}\right|,
\end{aligned}
$$

while the remaining parameters $q_{s}$, such as layer thicknesses, etc., are unchanged: $q_{s}=q_{j 0}$. We further assume that the $p_{\imath}$ are changed in this manner in all conditions where they may occur-in the differential equation as well as in the boundary conditions. This procedure has been applied in a convolution technique for generating wave solutions in absorptive structures from the solutions for their nonabsorptive counterpart [Shemyakin, 1955].

The frequencies and wave numbers $\omega$ and $k$ will be changed because of the perturbation according to (2). We set

$$
\begin{aligned}
& \omega=\omega_{0}+\omega_{1} \quad\left|\omega_{1}\right| \ll\left|\omega_{0}\right| \\
& k=k_{0}+k_{1} \quad\left|k_{1}\right| \ll\left|k_{0}\right|
\end{aligned}
$$

where both $k_{0}$ and $k_{1}$ are functions of $\omega$.

We expand (2) in a Taylor series about the lossless condition:

$$
\begin{aligned}
\omega_{0}+\omega_{1}=\omega\left(k_{0} ; p_{l 0}, q_{j 0}\right)+k_{1} \frac{\partial \omega_{0}}{\partial k} \\
+i \sum_{l=1}^{n} \frac{\partial \omega_{0}}{\partial p_{l}} p_{l 1}+\cdots
\end{aligned}
$$

where the symbol $\partial_{\omega_{0}} / \partial k$ is the value of the derivative $\partial \omega / \partial k$ for the lossless model. Retaining terms of the two lowest orders and applying
(3) we can write

$$
\omega_{1}=k_{1} \frac{\partial \omega_{0}}{\partial k}+i \sum_{l=1}^{n} p_{l 1} \frac{\partial \omega_{0}}{\partial p_{l}}
$$

Consider a standing wave experiment. At a single point of observation $x_{0}$, we approximate the observation of a damped harmonic oscillation by

$$
e^{-\alpha t} \sin (\omega t+\delta) \sin \left(k x_{0}+\phi\right)
$$

where $\alpha=\operatorname{Im} \omega$. In the case of standing waves $k$ is real and proportional to the reciprocal wavelength, a measurable quantity. Hence, $\operatorname{Im} k_{1}=0$. We assume further that Re $k \approx k_{0}$; because of causality, this approximation is in error at most by terms of order $1 / Q$. We can then write

$$
\alpha=\operatorname{Im} \omega_{1}=\operatorname{Re} \sum_{l=1}^{n} \frac{\partial \omega_{0}}{\partial p_{l}} p_{l 1}
$$

Now consider a second experiment performed upon propagating waves. At a fixed time $t_{0}$ the spatial distribution of the wave function can be approximated by the function

$$
e^{-\gamma x} \sin \left(\omega t_{0}-k x+\phi^{\prime}\right) \quad x>0
$$

where $-\gamma=\operatorname{Im} k_{1}$. In this case $\omega$ is real and proportional to the reciprocal period, a measurable quantity. Hence we take $\operatorname{Im} \omega_{1}=0$. It follows, then, that

$$
\frac{\partial \omega_{0}}{\partial k} \gamma=\operatorname{Re} \sum_{l=1}^{n} \frac{\partial \omega}{\partial p_{l}} p_{l 1}
$$

where $\partial \omega_{0} / \partial k$ is real by the assumptions of (2).

The quantity $U_{0}$, defined as $U_{0}=\partial \omega_{0} / \partial k$, has all the features of group velocity, but it is determined for a dissipationless model from the continuous function (2). It is clear, then, that $\alpha=U_{\circ} \gamma$.

Finally we approximate $U=\operatorname{Re} \partial \omega / \partial k \approx U_{0}$; that is, the group velocity for the model is equal to the group velocity in the absence of dissipation. Because of causality, this approximation is in error by at most terms of order $1 / Q$. Then

$$
\alpha=U_{\gamma}
$$

Thus we see that the measurements of $Q$ as defined from the logarithmic decrement in the two types of experiments lead to different values. In the standing wave experiment, we find a value $Q_{r}$ given by $\alpha=\omega /\left(2 Q_{r}\right)$ while in the 
propagating wave case we have a value $Q_{x}$ given by $\gamma=\omega /\left(2 c Q_{x}\right)$. By the relationship (7) equation 1 follows for large $Q$. Hence $Q_{T}$ 's measured from the observations of the free modes of oscillation of the earth must be modified when they are to be compared with $Q_{x}$ 's at the same periods obtained from propagating wave experiments.

\section{REFERENCES}

Brune, J. N., Attenuation of dispersed wave trains, Bull. Seismol. Soc. Am., 62, 109-112, 1962.

Futterman, W. I., Dispersive body waves, J. Geophys. Res., 67, 5279-5291, 1963.

Shemyakin, E. I., The Lamb problem for a medium with an elastic aftereffect, Dokl. Akad. Nauk SSSR, 104, 193-196, 1955.

(Received November 2, 1963.) 